\title{
Integrated bioinformatics analysis reveals novel hub genes closely associated with pathological mechanisms of immunoglobulin A nephropathy
}

\author{
DONGMEI ZHANG* , YILING CAO*, YONGDI ZUO, ZHENG WANG, XUHUA MI and WANXIN TANG
}

Department of Nephrology, West China Hospital, Sichuan University, Chengdu, Sichuan 610000, P.R. China

Received November 4, 2018; Accepted May 9, 2019

DOI: $10.3892 /$ etm.2019.7686

\begin{abstract}
Immunoglobulin A (IgA) nephropathy (IgAN) is the most common glomerular disease. The major pathological changes associated with it affect cell proliferation, fibrosis, apoptosis, inflammation and extracellular matrix (ECM) organization. However, the molecular events underlying IgAN remain to be fully elucidated. In the present study, an integrated bioinformatics analysis was applied to further explore novel potential gene targets for IgAN. The mRNA expression profile datasets GSE93798 and GSE37460 were downloaded from the Gene Expression Omnibus database. After data preprocessing, differentially expressed genes (DEGs) were identified. Gene Ontology (GO) enrichment analysis of DEGs was performed. Protein-protein interaction (PPI) networks of the DEGs were built with the STRING online search tool and visualized by using Cytoscape, and hub genes were identified through the degree of connectivity in the PPI. The hub genes were subjected to Kyoto Encyclopedia of Genes and Genomes pathway analysis, and co-expression analysis was performed. A total of 298 DEGs between IgAN and control groups were identified, and 148 and 150 of these DEGs were upregulated and downregulated, respectively. The DEGs were enriched in distinct GO terms for Biological Process, including cell growth, epithelial cell proliferation, ERK1 and ERK2 cascades, regulation of apoptotic signaling pathway and ECM organization. The top 10 hub genes were then screened from the PPI network by Cytoscape. As novel hub genes, Fos proto-oncogene, AP-1 transcription factor subunit and early growth response 1 were determined to be closely associated with apoptosis and cell proliferation in $\operatorname{Ig} \mathrm{AN}$. Tumor protein 53, integrin subunit $\beta 2$ and fibronectin 1
\end{abstract}

Correspondence to: Professor Wanxin Tang, Department of Nephrology, West China Hospital, Sichuan University, 37 Guoxue Alley, Chengdu, Sichuan 610000, P.R. China

E-mail: kidney123@163.com

*Contributed equally

Key words: bioinformatics, IgA nephropathy, gene expression, hub gene may also be involved in the occurrence and development of IgAN. Co-expression analysis suggested that these hub genes were closely linked with each other. In conclusion, the present integrated bioinformatics analysis provided novel insight into the molecular events and novel candidate gene targets of IgAN.

\section{Introduction}

Immunoglobulin A ( $\operatorname{Ig} \mathrm{A})$ nephropathy $(\operatorname{Ig} \mathrm{AN})$ is a common primary renal disease worldwide $(1,2)$ and has emerged as an important healthcare issue (3). Cell proliferation $(4,5)$, fibrosis $(6,7)$, apoptosis $(8,9)$ and sustained inflammation (10) are involved in the pathogenesis of IgAN. Inhibition of human mesangial cell proliferation by targeting $\mathrm{C} 3 \mathrm{a} / \mathrm{C} 5$ a receptors has been demonstrated to alleviate IgAN in mice (4). Glomerular endothelial proliferation has been reported to contribute to renal injury in $\operatorname{IgAN}(11,12)$. Cytotoxin-associated antigen A may induce cellular injury in glomerular mesangium through the proliferation and secretion of the extracellular matrix (ECM), which may have an important role in the pathogenesis of IgAN (13). Renal expression of microRNA (miR)-21-5p is associated with fibrosis and renal survival in patients with $\operatorname{IgAN}(6)$, and rapamycin may reduce apoptosis of podocytes under stimulated conditions of $\operatorname{IgAN}(8,9)$. However, the crucial genes involved in $\operatorname{IgAN}$ have remained elusive due to limited large-scale studies, and methods for the effective early diagnosis and treatment of $\operatorname{IgAN}$ remain unavaliable.

Bioinformatics analysis is a powerful research method used to predict molecular mechanisms and associations among genes. This approach has been used to predict novel genes and pathways associated with tumors, including hepatocellular carcinoma (14), non-small cell lung cancer (15), osteosarcoma (16) and esophageal adenocarcinoma (17). Bioinformatics analysis has gradually provided insight into the molecular mechanisms of kidney disease (18). For instance, the gene expression profile of macrophages was recently analyzed through a bioinformatics analysis, indicating the induction of CCL2 and CD38 in macrophages from patients with lupus nephritis (19). To date, only few bioinformatics analyses have been performed on IgAN. A distinct glomerular molecular signature associated with endocapillary proliferation has been identified in patients with IgAN through a gene expression 
profiling array (12). However, to the best of our knowledge, no integrated and in-depth data analysis associated with IgAN has been previously performed. Therefore, it is necessary to identify genes associated with IgAN through integrated bioinformatics analysis. In the present study, several gene expression profile datasets were downloaded from the Gene Expression Omnibus (GEO) database. After data integration processing, differentially expressed genes (DEGs) were identified, and Gene Ontology (GO) functional analysis was performed. The top upregulated and downregulated hub genes in five disease-associated biological functions were further analyzed. Protein-protein interaction (PPI) networks of the DEGs were built and the hub genes were identified. The present results indicated that these hub genes were involved in different pathological mechanisms in IgAN. The DEGs co-expressed with the top hub genes were then further analyzed. The present study aimed to discover potential novel candidate molecular targets in IgAN.

\section{Materials and methods}

Retrieval of IgAN-associated gene expression data. Human IgAN microarray datasets were searched and downloaded from the National Center for Biotechnology Information (NCBI) GEO database (http://www.ncbi.nlm.nih.gov/geo). The keyword 'IgA nephropathy' was used for accurate searching. The data selection criteria were as follows: i) All datasets were expression profiles, ii) all samples were kidney glomerular tissues, iii) the species was Homo sapiens, and iv) complete microarray raw data were available. Finally, two datasets, namely GSE93798 (20) and GSE37460 $(21,22)$, were finally selected on the basis of the abovementioned criteria with exclusion of the duplicate data, for integrated analysis. The integrated datasets included $47 \mathrm{IgAN}$ and 31 normal glomerular tissues. Original CEL files and platform probe annotation information files were subjected to further bioinformatics analysis.

Data preprocessing. The preprocessing and normalization of microarray datasets with raw data (.CEL files) were performed with the RMA function in the Affy package in the R environment (version 3.2.3) (23) with the following parameters: Data normalization using quantile normalization and background correction using RMA background correction with a background similar to the pure RMA background given in the Affy version 1.1 and above $(23,24)$. After the gene expression value was obtained, the 'annotate' software package was used to annotate the genes and the expression matrix was merged. The batch effects from the microarray were removed by the function Combat in the SVA package with the 'Empirical Bayes methods' (25).

$D E G$ analysis. The DEGs between the Ig AN and normal tissues were analyzed with the Limma package in $\mathrm{R}(26)$. The linear fit method (using the lmFit function with default options), Bayesian analysis (using the eBayes function with default options) and the t-test algorithm were utilized to calculate the P-values and fold change (FC) values. The TopTable function in the Limma package was used to screen the DEGs (parameters: Adjust. method='fdr', $\operatorname{coef}=1$, adjusted (adj.)P-value $=0.05, \operatorname{lfc}=\log (2,2)$, number $=5,000$, and sort.by=' $\left.\log F C^{\prime}\right)$. An adj.P-value $<0.05$ and $\log 2 \mathrm{FCl} \geq 1$ were set as the cutoff parameters to screen any significantly upregulated or downregulated genes. The ggplot2 software package was used to visualize the DEGs.

Functional enrichment analysis. The ClusterProfiler version 3.5 is an $\mathrm{R}$ package for the biological term classification and enrichment analysis of gene clusters (27). The cluster profiler package was used to perform GO functional enrichment analysis for the DEGs. Adj.P-value $<0.05$ was set as the cutoff criterion for $\mathrm{GO}$ enrichment analysis.

PPI network analysis and hub gene identification. The DEGs identified were subjected to PPI analysis by using the search functionality of STRING (http://string.embl.de/) (28) to explore the association between the DEGs, and a network interaction matrix was built. The minimum required interaction score of 0.7 was the cutoff threshold. The PPI network data matrix was downloaded for further analysis and visualization by using Cytoscape (version 6.3; http://www.cytoscape. org/) (29). CytoHubba (30) is a tool used to identify hub objects and subnetworks from a complex interactome. 'Degree' is a topological analysis method in CytoHubba. 'Degree' was used to discover featured nodes and identify the hub genes from all DEGs.

Kyoto Encyclopedia of Genes and Genomes (KEGG) pathway analysis. The top five hub genes in the five disease-associated GO terms were selected by consulting the literature in the NCBI database to find the potential KEGG pathway associations with IgAN. The KEGG tool (http://www.genome. $\mathrm{jp} / \mathrm{kegg} /$ pathway.html) was used to further analyze the signaling pathways of the selected target genes.

Co-expressed gene analysis. Pearson correlation coefficients between each top hub gene and all other DEGs were calculated by using the package 'Hmisc' (version 4.1.1). The top five significant genes associated with each selected top hub gene were screened. The co-expression association between each top hub gene and other DEGs was further analyzed using Cytoscape.

\section{Results}

Identification of DEGs. A total of 78 samples, comprising 47 IgAN samples and 31 normal samples, in the two datasets were included for analysis in the present study. The detailed information of all of the samples is listed in Table SI. Based on the cutoff criteria of $\log 2 \mathrm{FCl} \geq 1.0$ and adj.P-value $\leq 0.05$, 298 DEGs in the IgAN group vs. control group were obtained. Among those DEGs, 148 and 150 genes were upregulated and downregulated, respectively. The results on the expression level analysis are presented in a volcano plot in Fig. 1A. As indicated in the graph, the data distribution of the upregulated, downregulated or insignificantly changed gene expression levels was normal. Transcriptional and immune response regulator, fatty acid binding protein 5 , tropomyosin 1 , colony-stimulating factor 1 receptor, erythrocyte membrane protein band 4.1 like 2 (EPB41L2), fibronectin 1 (FN1), hemoglobin subunit $\beta, \mathrm{ECM}$ protein 1 , adipocyte enhancer-binding 
A

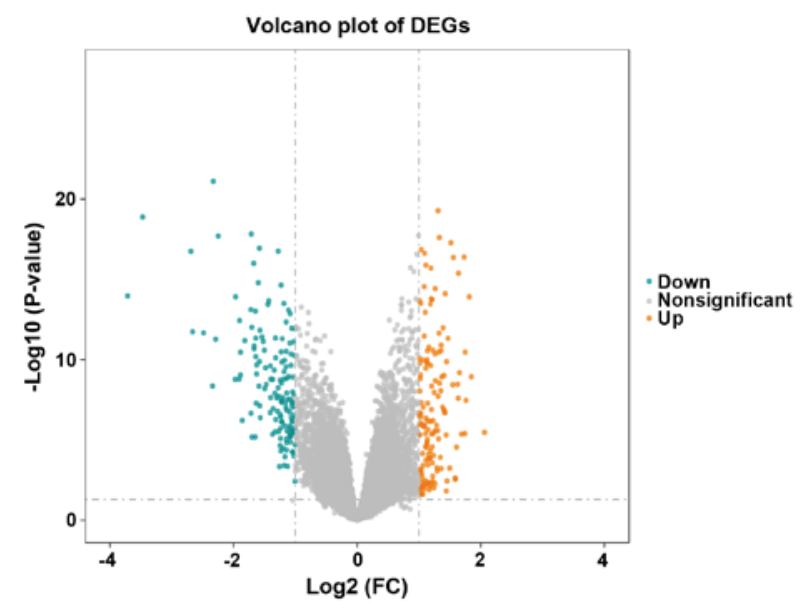

B

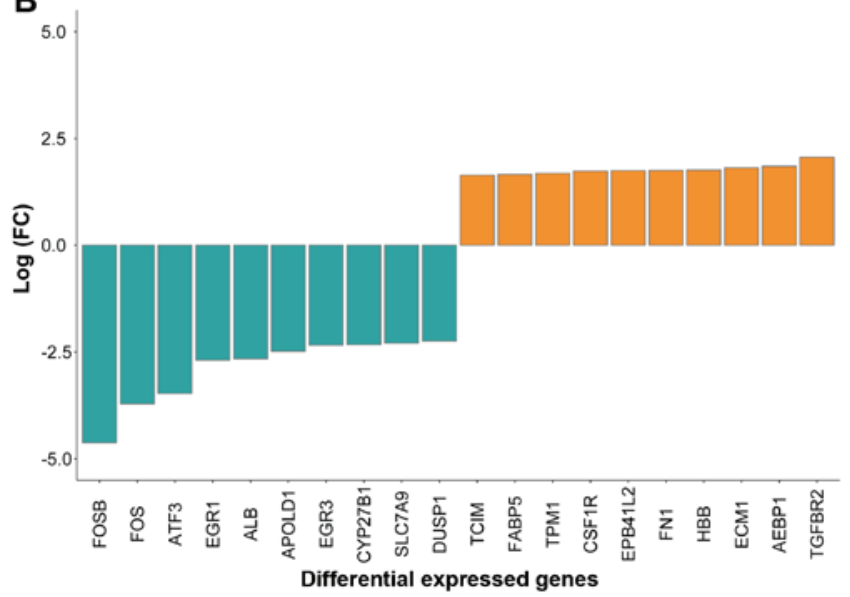

Figure 1. Expression patterns of DEGs. (A) Volcano plot of all DEGs. Orange dots indicate high expression levels in IgAN tissues, whereas blue dots denote low expression levels. Gray dots correspond to the genes with a $\log 2 \mathrm{Fcl}<1$ or adj.P-value $>0.05$. The dots in the area above the horizontal dotted line have an adj.P-value $<0.05$. The dots outside the two vertical dotted lines have a $\mid \log 2 \mathrm{Fcl} \geq 1$. (B) Top 10 upregulated and downregulated DEGs in IgAN $\mid \log 2 \mathrm{Fcl}>1$ and adj.P-value $<0.05$ were set as the selection criteria. $\log (\mathrm{FC})$ $>0$, upregulated; $\log (\mathrm{FC})<0$, downregulated. DEG, differentially expressed gene; adj., adjusted; FC, fold change; IgAN, immunoglobulin A nephropathy; FOSB, FosB proto-oncogene, AP-1 transcription factor subunit; ATF3, activating transcription factor 3; EGR1, early growth response 1; ALB, albumin; APOLD1, apolipoprotein L domain containing 1; CYP27B1, cytochrome P450 family 27 subfamily B member 1; SLC7A9, solute carrier family 7 member 9; DUSP1, dual specificity phosphatase 1; TCIM, transcriptional and immune response regulator; FABP, fatty acid binding protein; TPM1, tropomyosin 1; CSF1R, colony-stimulating factor 1 receptor; EPB41L2, erythrocyte membrane protein band 4.1 like 2; FN1, fibronectin 1; HBB, hemoglobin subunit $\beta$; ECM1, extracellular matrix protein 1; AEBP, adipocyte enhancer-binding protein; TGFBR2, transforming growth factor $\beta$ receptor 2 .

protein and transforming growth factor $\beta$ receptor 2 (TGFBR2) were the 10 most significantly upregulated genes in IgAN, whereas FosB proto-oncogene, AP-1 transcription factor subunit (FOSB), FOS, activating transcription factor 3 (ATF3), early growth response 1 (EGR1), albumin (ALB), apolipoprotein L domain containing 1, EGR3, cytochrome P450 family 27 subfamily B member 1 (CYP27B1), solute carrier family 7 member 9 and dual specificity phosphatase 1 (DUSP1) were the 10 most substantially downregulated genes (Fig. 1B). Additional detailed information on all DEGs is listed in Table SII.
PPI network and hub gene analysis. In the PPI network analysis, the average node degree of connectivity was 2.57 , the average local clustering coefficient was 0.366 and the number of edges was 172. The P-value for clusters of interacted proteins was $<1.0 \times 10^{-16}$. Finally, 10 top-ranked hub genes were identified from all the DEGs in the network by Degree analysis, as presented in Fig. 2A (colored nodes). These genes were tumor protein (TP53), integrin subunit $\beta 2$ (ITGB2), FN1, FOS, complement C3a receptor 1 (C3AR1), EGR1, ALB, Fc fragment of IgE receptor Ig (FCER1G), phosphatidylinositol-4,5-bisphosphate 3-kinase catalytic subunit $\alpha$ (PIK3CA) and SHC adaptor protein 1 (SHC1). In the network, these hub genes had higher Degree scores than the other hub genes (Fig. 2A). The genes were closely correlated with one another. Among the 10 hub genes, seven genes, including TP53, ITGB2, FN1, C3AR1, FCER1G, PIK3CA and SHC1, were upregulated, and three genes, including FOS, EGR1 and ALB, were downregulated (Fig. 2B). Of note, among the top 10 hub genes, ALB (31), FOS (32) and TP53 (33) are known to be involved in the pathological process of IgAN. The top five hub genes were considered for subsequent KEGG pathway analysis to narrow down the analysis.

Co-expressed gene analysis. Pearson correlation analysis revealed the major DEGs co-expressed with TP53, ITGB2, FN1, FOS, C3AR1, EGR1, ALB, FCER1G, PIK3CA and SHC1 (Fig. 2C). The top five DEGs co-expressed with TP53 were EPB41L2, SWI/SNF related, matrix associated, actin-dependent regulator of chromatin, subfamily A, member 4, MIS18 binding protein 1, FKBP prolyl isomerase $1 \mathrm{~A}$ and $\mathrm{SHC}$. The genes co-expressed with ITGB2 were neutrophil cytosolic factor 2, C3AR1, FCER1G, TYRO protein tyrosine kinase binding protein and lysosomal protein transmembrane 5 . Collagen type I $\alpha 2$ chain, TGFB1, periostin, transgelin and complement C1q A chain were closely associated with FN1. Of these, ITGB2 is possibly involved in the pathological process of IgAN through ECM remodeling and apoptosis $(34,35)$, whereas FN1 may be implicated in IgAN-associated fibrosis (36). Additional information on the top six novel hub genes is listed in Table I (37-69).

KEGG pathway analysis of hub genes. First, Homo sapiens was selected as the organism. TP53, ITGB2, FN1, FOS and C3AR1 were then entered in the keyword dialog box. The pathways involved in cell cycle and proliferation, inflammation, apoptosis and focal adhesion were selected for presentation. TP53 was indicated to be involved in the P53 signaling pathway, which is associated with the cell cycle, apoptosis and inhibition of metastasis (Fig. 3A). ITGB2 was indicated to participate in the HIPPO signaling pathway, which regulates the expression of anti-apoptotic genes, and is also associated with focal adhesion (Fig. 3B). FN1 and ITGB2 are implicated in focal adhesion through the same signaling pathway. In addition, FN1 was indicated to participate in apoptosis and mesangial matrix expansion (Fig. 3C). FOS was also suggested to be involved in apoptosis (Fig. 3D). All of these pathways are closely linked to IgAN. However, the search in the KEGG pathway database failed to identify any signaling pathway where C3AR1 is directly involved in the cell cycle and proliferation, inflammation, apoptosis and focal adhesion. 


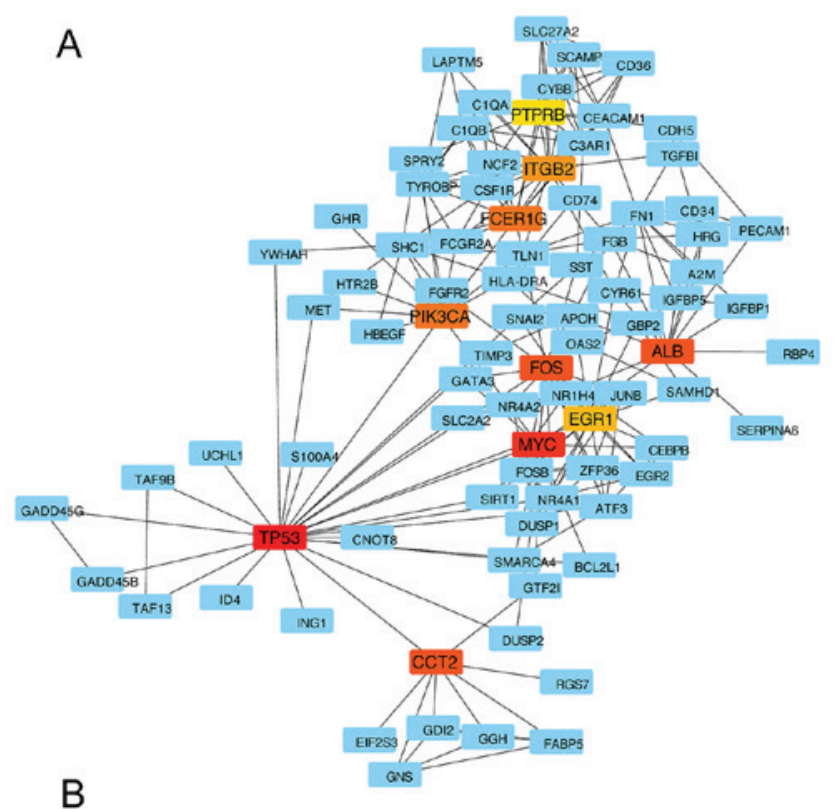

\begin{tabular}{|c|c|c|}
\hline Rank & Name & Score \\
\hline 1 & TP53 & 25 \\
\hline 2 & ITGB2 & 20 \\
\hline 3 & FN1 & 18 \\
\hline 4 & FOS & 16 \\
\hline 4 & C3AR1 & 16 \\
\hline 6 & EGR1 & 14 \\
\hline 6 & ALB & 14 \\
\hline 8 & FCER1G & 13 \\
\hline 8 & PIK3CA & 13 \\
\hline 10 & SHC1 & 11 \\
\hline
\end{tabular}
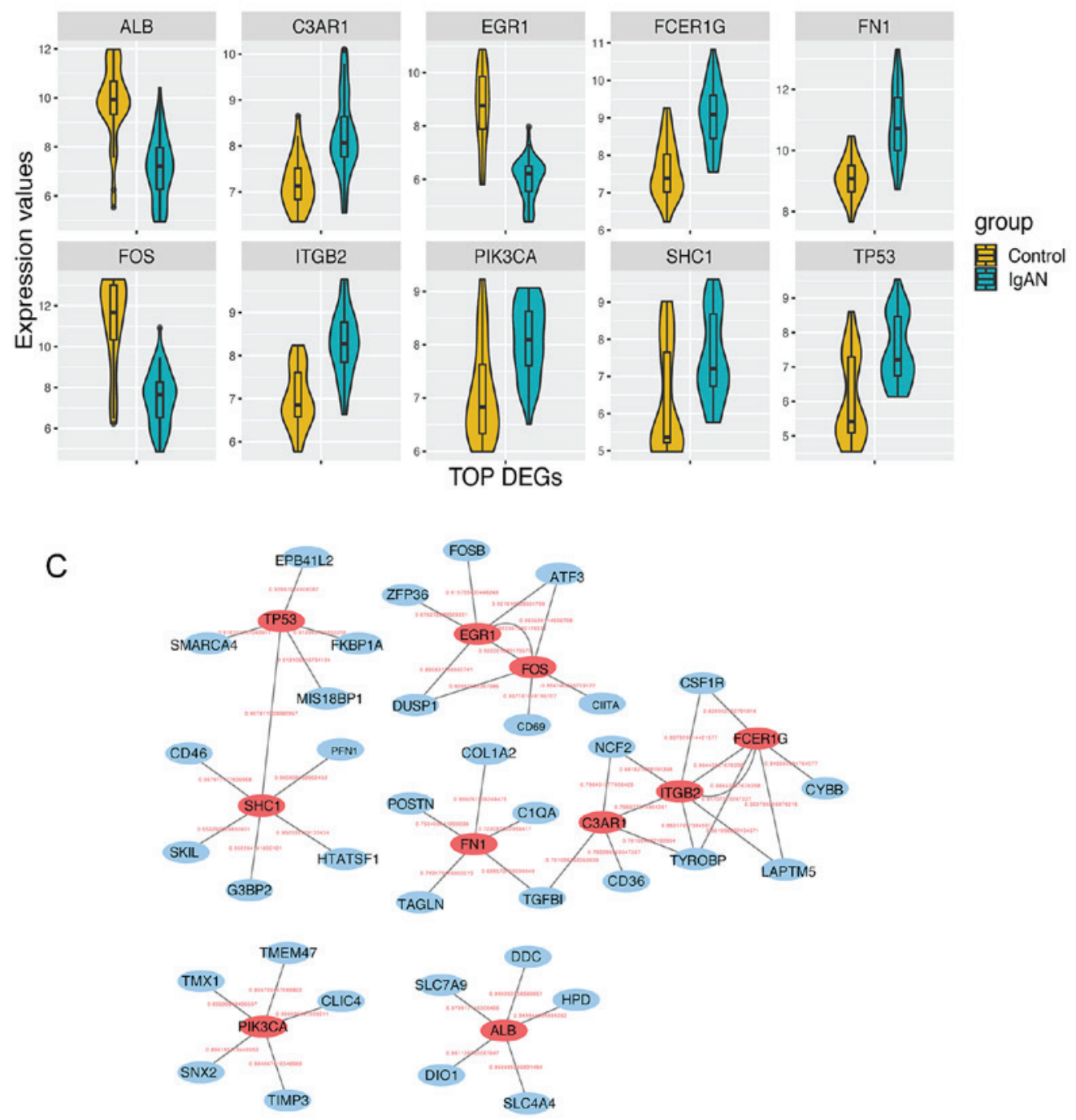

Figure 2. (A) PPI network and top hub genes. Hub genes were identified from all DEGs by using the Degree analysis method. The depth of colour from blue to red indicates the rank from low to high of the hub genes. The table on the right-hand side presents the rank scores by Degree of the top 10 hub genes. (B) Expression patterns of the top 10 hub genes screened out from the PPI networks between the control group and the IgAN groups. (C) One cluster represents the top five gene ontology terms co-expressed with a hub gene (red nodes). The red digits indicate correlation coefficients. All P-values of the correlation are $<0.05$. Regarding the definition of all gene names, please refer to Table SIV. PPI, protein-protein interaction; DEG, differentially expressed gene; IgAN, immunoglobulin A nephropathy; TP53, tumor protein 53; FOS, Fos proto-oncogene, AP-1 transcription factor subunit; EGR1, early growth response 1; ALB, albumin; ITGB2, integrin subunit $\beta 2$; FN, fibronectin; C3AR, complement C3a receptor 1; FCER1G, Fc fragment of IgE receptor Ig; PIK3CA, phosphatidylinositol-4,5-bisphosphate 3-kinase catalytic subunit $\alpha$; SHC1, SHC adaptor protein 1. 
Table I. Extended information on the function of the top 6 hub genes.

\begin{tabular}{|c|c|c|c|}
\hline Gene & Function & Associated pathway & (Refs.) \\
\hline \multirow[t]{6}{*}{ TP53 } & $\begin{array}{l}\text { Deregulation of TP53 in deregulation of } \\
\text { TP53 in multiple myeloma }\end{array}$ & Tp53 pathway & $(37)$ \\
\hline & Tumor suppressive & DNA damage-induced apoptosis & $(38)$ \\
\hline & Regulated inhibitor of apoptosis & Regulating podocyte apoptosis & $(39)$ \\
\hline & $\begin{array}{l}\text { Contributes to the pathogenesis of dilated } \\
\text { cardiomyopathy }\end{array}$ & DNA damage response/TP53 pathway & $(40)$ \\
\hline & Increases susceptibility to cervical cancer development & $\begin{array}{l}\text { Interaction between the XRCC1 and TP53 } \\
\text { genes }\end{array}$ & $(41)$ \\
\hline & Response to stress & Autophagy and apoptosis & $(42)$ \\
\hline \multirow[t]{5}{*}{ ITGB2 } & Inhibits osteosarcoma proliferation and metastasis & Wnt/ $\beta$-catenin signalling & (43) \\
\hline & Mediates cell invasion & Leukocyte-specific integrin $\beta 2$ expression. & $(44)$ \\
\hline & Promotes macrophage retention & Inflammatory & $(45)$ \\
\hline & Causes canine leukocyte adhesion deficiency & Missense mutation & $(46)$ \\
\hline & Inhibits TLR responses & NF- $\kappa$ B pathway and p38 MAPK activation. & $(47)$ \\
\hline \multirow[t]{4}{*}{ FN1 } & Promotes apoptosis of epithelial cells & MiR-206 /FN1 & $(48)$ \\
\hline & Suppresses apoptosis & $\mathrm{NF}-\kappa \mathrm{B}$ pathway & (49) \\
\hline & $\begin{array}{l}\text { Reverses the radioactive iodine resistance of } \\
\text { papillary thyroid carcinoma cell }\end{array}$ & $\begin{array}{l}\text { MiR-101-3p/FN1/PI3K/AKT signaling } \\
\text { pathway }\end{array}$ & $(50)$ \\
\hline & Cell proliferation, senescence and apoptosis & PI3K/AKT signaling pathway & $(51)$ \\
\hline \multirow[t]{6}{*}{ FOS } & Chronic inflammation & Metabolic pathways & $(52)$ \\
\hline & Inflammation & AP-1 and AKT/mTor pathways & $(53)$ \\
\hline & Inflammatory injury & $\begin{array}{l}\text { Oxidative stress-mediated FOS/IL8 } \\
\text { signaling }\end{array}$ & $(54)$ \\
\hline & Regulates cell cycle & P38 MAPK/AP-1 factors & $(55)$ \\
\hline & Proliferation and apoptosis of hippocampal neurons & MAPK signaling pathway & $(56)$ \\
\hline & Regulates cervical cancer cells growth & ERK1/2/c-Fos/c-Jun & $(57)$ \\
\hline \multirow[t]{4}{*}{ C3AR1 } & VEGFR2 survival and mitotic signaling & C3AR1/C5AR1 and IL-6R-GP130 & $(58)$ \\
\hline & $\begin{array}{l}\text { Limits expansion and differentiation of alloreactive } \\
\mathrm{CD} 8^{+} \mathrm{T} \text {-cell immunity }\end{array}$ & C3AR1 signaling & (59) \\
\hline & Enhances the formation of intestinal organoids & C3AR1 signaling & $(60)$ \\
\hline & Up-regulated genes in T2DM & Type 2 diabetes mellitus & (61) \\
\hline \multirow[t]{8}{*}{ EGR 1} & Proliferation and fibrosis & TGF- $\beta 1$ signaling & $(62)$ \\
\hline & Prevents renal tubulointerstitial fibrosis & MiR-192/TGF- $\beta 1 / \mathrm{FN}$ & $(63)$ \\
\hline & $\begin{array}{l}\text { Reducing the expression of fibrosis and } \\
\text { inflammatory cytokines }\end{array}$ & EGR1/TLR4/mTor axis & (64) \\
\hline & Transcriptional activator of NOX4 & Oxidative stress & $(65)$ \\
\hline & Renal fibrosis & TGF- $\beta 1 /$ Smad3 signaling & (66) \\
\hline & Tumor angiogenesis regulation & $\begin{array}{l}\text { MiR-192/EGR1/HOXB9 regulatory } \\
\text { network }\end{array}$ & (67) \\
\hline & $\begin{array}{l}\text { Mediates chronic hypoxia-induced renal interstitial } \\
\text { fibrosis }\end{array}$ & PKC/ERK pathway. & $(68)$ \\
\hline & Renal epithelial cell migration and apoptosis & GDNF/EGR1 pathway & (69) \\
\hline
\end{tabular}

TP53, tumor protein 53; FN, fibronectin; ECM, extracellular matrix; FOS, Fos proto-oncogene, AP-1 transcription factor subunit; ITGB2, integrin subunit $\beta 2$; EGR, early growth response; C3AR, complement C3a receptor 1; miR, microRNA; PKC, protein kinase C; TLR, Toll-like receptor; TGF, transforming growth factor; IL, interleukin; XRCC1, X-ray repair cross complementing 1; VEGFR, vascular endothelial growth factor receptor; NOX4, NADPH oxidase 4.

GO enrichment. The ClusterProfiler package was used for pathway enrichment analysis and GO analysis to reveal the biological functions based on the DEGs. The 15 most significant
GO terms in the category biological process from the groups with adj.P $<0.05$ are presented in Fig. 4A and the 15 most significant GO terms in the category molecular function from 
A hsa04115

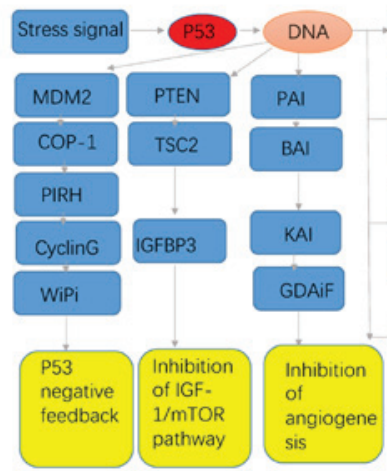

P53 signaling pathway

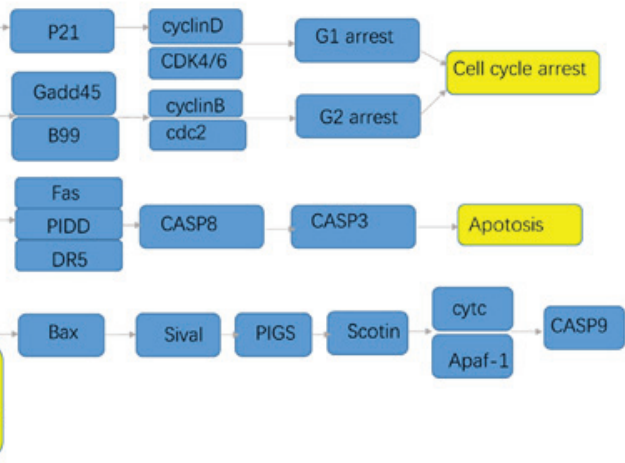

B hsa04390,hsa04933,and hsa05133 HIPPO signaling pathway
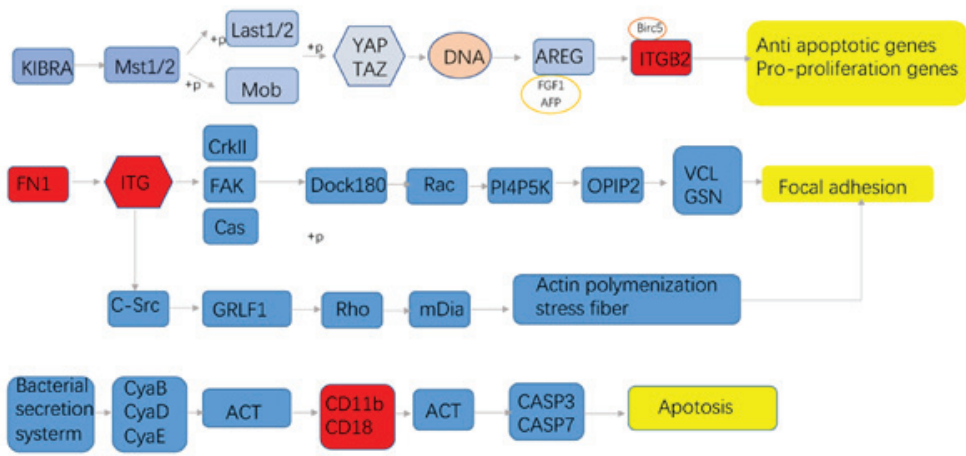

C hsa04151,has04510,and hsa04933 PI3K-AKT signaling pathway

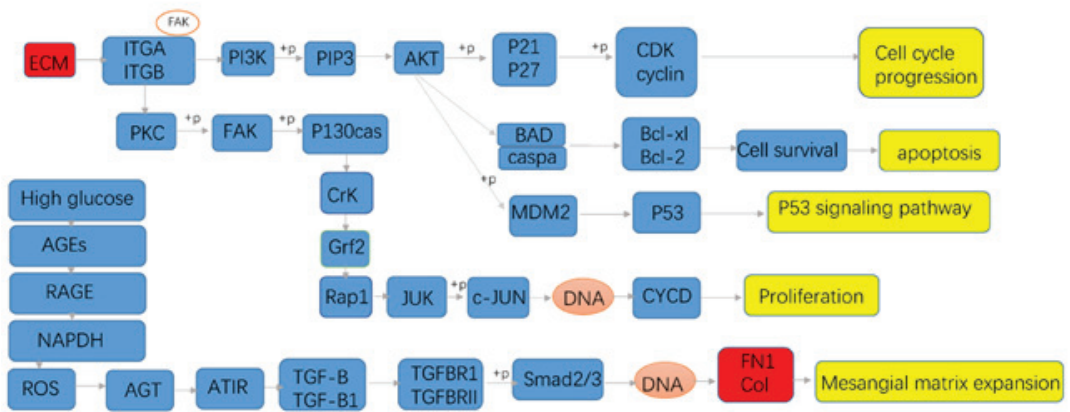

D

hsa01522, hsa04010,hsa04310, and hsa04657

IL-17 signaling pahway

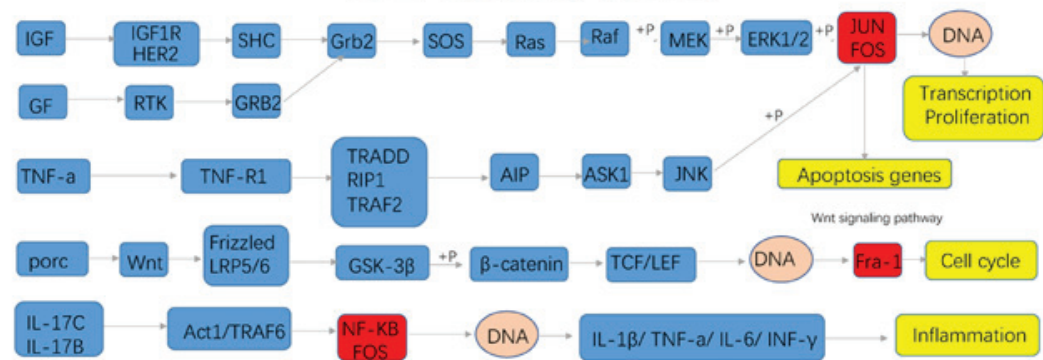

Figure 3. KEGG pathway analysis for hub genes. (A) TP53 was significantly involved in the P53 signaling pathway. This figure was redrawn on the basis of the KEGG pathway hsa04115. (B) ITGB2 was indicated to participate in the HIPPO signaling pathway. This figure was redrawn on the basis of the KEGG pathways hsa04390, hsa04933 and hsa05133. (C) FN1 was indicated to be significantly involved in the ECM/PI3K/AKT signaling and mesangial matrix expansion pathways. This figure was redrawn on the basis of the KEGG pathways hsa04151, has04510 and hsa04933. (D) FOS was indicated to participate in the Wnt signaling, IL-17 signaling and apoptosis pathways. This figure was redrawn on the basis of the KEGG pathways hsa01522, hsa04010, hsa04310 and hsa04657. Regarding the definition of all gene names, please refer to Table SIV. KEGG, Kyoto Encyclopedia of Genes and Genomes; hsa, Homo sapiens; TP53, tumor protein 53; IL, interleukin; FN, fibronectin; ECM, extracellular matrix; FOS, Fos proto-oncogene, AP-1 transcription factor subunit; ITGB2, integrin subunit $\beta 2$. 
A

B
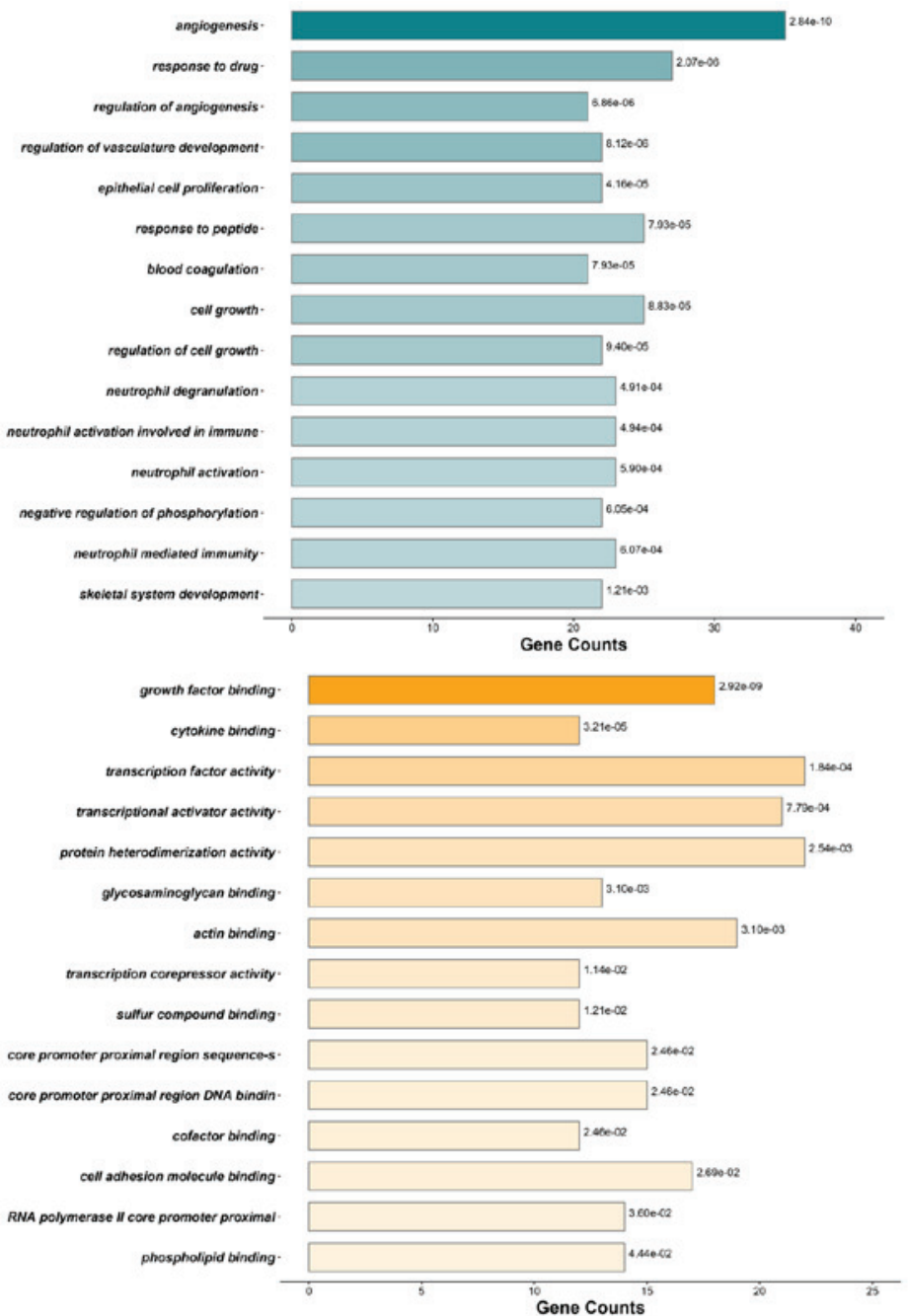

C

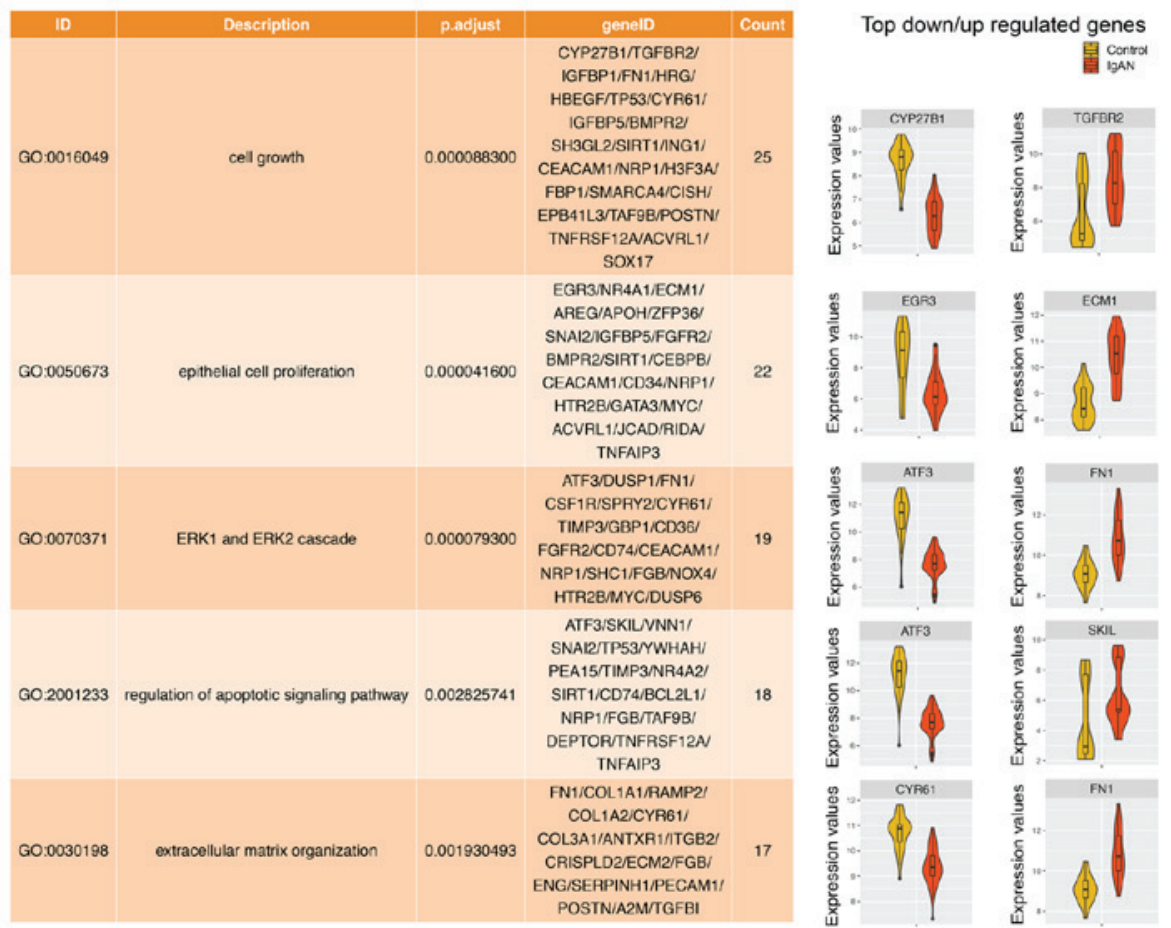

Figure 4. GO enrichment results of DEGs. (A) GO terms in the category biological process enriched by the DEGs. (B) GO terms in the category molecular function enriched by the DEGs. An adj.P-value $<0.05$ was used as the cut-off criterion. The length of each bar represents the gene counts in the GO term. The adj.P-value of each GO term is printed on the right side of the bars. (C) Five GO terms closely associated with the pathological mechanisms of IgAN. (D) Top upregulated and downregulated hub genes in each GO term. Regarding the definition of all gene names, please refer to Table SIV. GO, gene ontology; adj., adjusted; DEG, differentially expressed gene; IgAN, immunoglobulin A nephropathy. 
the groups with adj.P $<0.05$ are presented in Fig. 4B. The five GO terms closely associated with pathological mechanisms are provided in Fig. 4C. A total of 25 DEGs were involved in cell growth (GO:0016049), and 22 DEGs were implicated in the regulation of cell growth and epithelial cell proliferation (GO:0050673). Furthermore, 19 DEGs participated in the ERK1 and ERK2 cascades (GO:0070371) and 18 DEGs functioned in the regulation of the apoptotic signaling pathway (GO:2001233). In addition, 17 DEGs had a role in the ECM organization (GO:0030198). The top upregulated and downregulated hub genes in these disease-associated processes and functions are provided in Fig. 4D. CYP27B1, EGR3, ATF3, ATF3 and cysteine-rich angiogenic inducer 61 (CYR61) were the top downregulated hub genes in the five GO terms closely associated with pathological mechanisms, and TGFBR2, ECM3, FN1, SKI-like proto-oncogene and FN1 were the top upregulated hub genes in the five GO terms. All of the significantly enriched GO terms are listed in Table SIII.

\section{Discussion}

In the present study, 148 upregulated and 150 downregulated DEGs were identified from microarray data by applying an integrated bioinformatics analysis to further elucidate the molecular pathology of IgAN. GO analysis revealed that the DEGs were significantly enriched in cell growth, epithelial cell proliferation, ERK1 and ERK2 cascade, apoptotic signaling pathway regulation and ECM organization. CYP27B1 and TGFBR2 were enriched in the GO terms associated with cell growth. miR-195 was reported to inhibit proliferation, invasion and metastasis by targeting CYP27B1 in breast cancer cells (70), and CYP27B1 is involved in the anti-proliferative effects of 25-hydroxyvitamin D (71). miR-9-5p has been demonstrated to promote cell growth and metastasis in non-small cell lung cancer through repression of TGFBR2 (72). Cell proliferation is a vital factor in the pathogenesis of IgAN. For instance, circulating galactose-deficient IgA forms immune complexes deposited in the glomerular mesangium and causes local proliferation in IgAN (73). The present analysis revealed that ATF3 was enriched in GO terms including ERK1 and ERK2 cascades and regulation of the apoptotic signaling pathway. Cell migration and invasion may be strengthened by ATF3 through the activation of the p53 signaling pathway (74). Uremic toxins have been indicated to induce ATF3/c-Jun complex-mediated cannabinoid receptor type 1 expression by modulating the ERK1/2 and JNK signaling pathways and reactive oxygen species (75). FN1 was enriched in GO terms including the ERK1 and ERK2 cascades and the ECM organization. Depletion of FN1 was reported to markedly reduce the invasive capacity of prostate cancer cells (76). Furthermore, increased expression of FN1 in tumors may alter the primary tumor architecture, resulting in decreased metastasis formation (36). Treatment of PC-3 cells with $1 \mu \mathrm{M}$ FN1 was observed to result in a decrease in activated ERK1/2 (77). CYR61, which is also named cellular communication network factor 1 (CCN1), is an ECM-associated matricellular protein and one of the six members of the CCN family $(78,79)$. It may impair fibroblast responsiveness to TGF- $\beta$ signaling and upregulation of matrix metalloproteinase 1 (80). Fibrosis is closely associated with $\operatorname{IgAN}(6,7)$. Overall, the above indicates that the results of the functional analysis of the identified DEGs in the present study are reasonable and consistent with mechanisms identified by previous studies.

Several hub genes were identified from the PPI network, and this result is consistent with previously described genes, including ALB (31),FOS (32) and TP53 (33).TP53/p53 is a known regulator of apoptosis and macro-autophagy/autophagy (39). The coupled induction of inducible nitric oxide synthase and upregulation of TP53 in intrinsic renal cells of IgAN may be linked to pro- and anti-apoptotic activities (33). The present analysis revealed relatively few novel hub genes with a close association with the pathological processes of $\operatorname{IgAN}$, offering novel insight. ITGB2 is a receptor of intercellular adhesion molecule (ICAM)1, ICAM2, ICAM3 and ICAM4, and it is also called CD18. ITGB2 is involved in cellular adhesion and ECM remodeling in patients with renal cancer (34). Furthermore, ITGB2 was identified to be closely associated with apoptosis in patients with Alzheimer's disease (81). However, to the best of our knowledge, no previous study has reported on the role of ITGB2 in IgAN. In the present study, ITGB2 was the second-ranked hub gene in the PPI network. The KEGG analysis results confirmed that ITGB2 was directly involved in apoptosis and focal adhesion. Collectively, the novel hub gene ITGB2 was indicated to have an important role in IgAN.

Another novel and noteworthy hub gene identified in the present analysis is EGR1, a zinc finger transcription factor with an essential role in cell growth and proliferation (65). EGR1 contributes to diabetic kidney disease by enhancing epithelial-mesenchymal transition (65). Specific inhibition of EGR1 was observed to prevent mesangial cell hypercellularity in experimental nephritis (82). EGR1 overexpression in rhabdomyosarcoma significantly decreases cell proliferation, mobility and anchorage-independent growth (83). However, no previous study has reported on the role of EGR1 in IgAN. In the present study, EGR1 was among the top 10 hub genes in the PPI network. The present co-expression analysis indicated a close association between EGR1 and FOS. The expression levels of these two DEGs were decreased, possibly leading to a reduction in the inhibition of cell proliferation and resulting in the progression of IgAN.

The present study did not identify any direct significant GO term for 'fibrosis'. Renal biopsy in patients with IgAN is generally performed in the early stages of the disease, when renal fibrosis is not prominent. The GSE37460 dataset did not provide any clinical information. However, the GSE93798 dataset suggested that most of the patients' chronic kidney disease grades were below 3a and the Oxford Classification scores were relatively low, indicating that these patients were in the early stages of the disease (20). Therefore, abnormal expression of fibrosis-associated genes was not common in these samples. However, DUSP1, a gene associated with fibrosis, was among the DEGs. In chronic hypertension, angiotensin-1-7 increased DUSP1 to decrease fibrosis in resistance arterioles and attenuate end-stage organ damage (84). In the present study, the downregulation of DUSP1 may have acted as a fibrotic factor and prompt the onset of fibrosis.

The present study was the first to identify novel molecular targets by integrating all microarray datasets of IgAN in GEO. Thereby, the sample size was expanded and further information was obtained. The microarray matrix of the expression 
values was combined and the batch effects were removed by using the empirical Bayes method to make the data more comparable (85). The novel results may enhance the current understanding of the molecular pathogenesis of $\operatorname{IgAN}$. However, the present study has certain limitations. First, the clinical data from the GEO database were not available for each sample. Furthermore, the array data came from typical IgAN in the early stage, and therefore, the expression levels of certain genes may not be identical to those in the later stage. For instance, the protein levels of IGFBP1 have been reported to be upregulated in this disease (86), while this gene was downregulated in the present study. The cause of the inconsistency may be that it is unrealistic to dynamically obtain kidney tissue from a patient at different time-points. In addition, the novel potential candidate targets should be further validated in experimental studies. The present results were obtained using a bioinformatics screening to identify several novel DEGs between IgAN and healthy control samples, and suggested that part of the top hub genes have vital roles in the pathological process of cell proliferation in IgAN. Of note, the information provided in the present study was not limited to the top $10 \mathrm{hub}$ genes, but included certain other representative DEGs. The present results provide a valuable resource for future research on IgAN.

In conclusion, the present study was the first to apply an integrated bioinformatics analysis to investigate novel candidate genes and mechanisms involved in the pathogenesis of IgAN. ITGB2, FN1, ATF3 and EGR1 genes may have important roles in the development of $\operatorname{IgAN}$ and act as potential candidate molecular targets for the diagnosis and treatment of IgAN.

\section{Acknowledgements}

Not applicable.

\section{Funding}

This work was supported by the National Natural Science Foundation of China (grant no. 81270805) and the Science and Technology Department of Sichuan Province (grant no. 2018SZ0378).

\section{Availability of data and materials}

All data generated or analyzed during this study are available from the corresponding author on reasonable request.

\section{Authors' contributions}

WT designed the experiments; WT and DZ analyzed the data; YC, YZ, ZW and XM made substantial contributions to the acquisition of data wrote the manuscript. All authors read and approved the final manuscript.

\section{Ethics approval and consent to participate}

This research used public microarray data from the GEO database for bioinformatics analysis and did not involve any human participants.

\section{Patient consent for publication}

Not applicable.

\section{Competing interests}

The authors declare that they have no competing interests.

\section{References}

1. Wyatt RJ and Julian BA: IgA nephropathy. N Engl J Med 368: 2402-2414, 2013

2. Rodrigues JC, Haas M and Reich HN: IgA nephropathy. Clin J Am Soc Nephrol 12: 677-686, 2017.

3. Schena FP: A retrospective analysis of the natural history of primary IgA nephropathy worldwide. Am J Med 89: 209-215, 1990.

4. Zhang Y, Yan X, Zhao T, Xu Q, Peng Q, Hu R, Quan S, Zhou Y and Xing G: Targeting C3a/C5a receptors inhibits human mesangial cell proliferation and alleviates immunoglobulin A nephropathy in mice. Clin Exp Immunol 189: 60-70, 2017.

5. Rops A, Jansen E, van der Schaaf A, Pieterse E, Rother N, Hofstra J, Dijkman HBPM, van de Logt AE, Wetzels J, van der Vlag J and van Spriel AB: Interleukin-6 is essential for glomerular immunoglobulin A deposition and the development of renal pathology in Cd37-deficient mice. Kidney Int 93: 1356-1366, 2018.

6. Hennino MF, Buob D, Van der Hauwaert C, Gnemmi V, Jomaa Z, Pottier N, Savary G, Drumez E, Noël C, Cauffiez C and Glowacki F: miR-21-5p renal expression is associated with fibrosis and renal survival in patients with IgA nephropathy. Sci Rep 6: 27209, 2016.

7. Tanaka K, Sugiyama H, Yamanari T, Mise K, Morinaga H, Kitagawa M, Onishi A, Ogawa-Akiyama A, Tanabe K, Eguchi J, et al: Renal expression of trefoil factor 3 mRNA in association with tubulointerstitial fibrosis in $\operatorname{IgA}$ nephropathy. Nephrology (Carlton) 23: 855-862, 2018.

8. Liang S, Jin J, Lin B, Gong J, Li Y and He Q: Rapamycin induces autophagy and reduces the apoptosis of podocytes under a stimulated condition of immunoglobulin a nephropathy. Kidney Blood Press Res 42: 177-187, 2017.

9. Leung JC, Chan LY, Saleem MA, Mathieson PW, Tang SC and Lai KN: Combined blockade of angiotensin II and prorenin receptors ameliorates podocytic apoptosis induced by $\operatorname{IgA-activated}$ mesangial cells. Apoptosis 20: 907-920, 2015.

10. Rauen T and Floege J: Inflammation in IgA nephropathy. Pediatr Nephrol 32: 2215-2224, 2017.

11. Bao H, Chen H, Zhu X, Zhang M, Yao G, Yu Y, Qin W, Zeng C, Zen K and Liu Z: MiR-223 downregulation promotes glomerular endothelial cell activation by upregulating importin $\alpha 4$ and $\alpha 5$ in IgA nephropathy. Kidney Int 85: 624-635, 2014.

12. Hodgin JB, Berthier CC, John R, Grone E, Porubsky S, Gröne HJ, Herzenberg AM, Scholey JW, Hladunewich M, Cattran DC, et al: The molecular phenotype of endocapillary proliferation: Novel therapeutic targets for IgA nephropathy. PLoS One 9: e103413, 2014.

13. Wang L, Tan RZ, Chen Y, Wang HL, Liu YH, Wen D and Fan JM: CagA promotes proliferation and secretion of extracellular matrix by inhibiting signaling pathway of apoptosis in rat glomerular mesangial cells. Ren Fail 38: 458-464, 2016.

14. Cui H, Zhang Y, Zhang Q, Chen W, Zhao H and Liang J: A comprehensive genome-wide analysis of long noncoding RNA expression profile in hepatocellular carcinoma. Cancer Med 6: 2932-2941, 2017

15. Tian W, Liu J, Pei B, Wang X, Guo Y and Yuan L: Identification of miRNAs and differentially expressed genes in early phase non-small cell lung cancer. Oncol Rep 35: 2171-2176, 2016.

16. Dong B, Wang G, Yao J, Yuan P, Kang W, Zhi L and He X: Predicting novel genes and pathways associated with osteosarcoma by using bioinformatics analysis. Gene 628: 32-37, 2017.

17. Liu D: LYN, a key gene from bioinformatics analysis, contributes to development and progression of esophageal adenocarcinoma. Med Sci Monit Basic Res 21: 253-261, 2015.

18. Zhou LT, Qiu S, Lv LL, Li ZL, Liu H, Tang RN, Ma KL and Liu BC: Integrative bioinformatics analysis provides insight into the molecular mechanisms of chronic kidney disease. Kidney Blood Press Res 43: 568-581, 2018. 
19. Shu B, Fang Y, He W, Yang J and Dai C: Identification of macrophage-related candidate genes in lupus nephritis using bioinformatics analysis. Cell Signal 46: 43-51, 2018.

20. Liu P, Lassen E, Nair V, Berthier CC, Suguro M, Sihlbom C, Kretzler M,Betsholtz C, Haraldsson B, Ju W, et al: Transcriptomic and proteomic profiling provides insight into mesangial cell function in IgA nephropathy. J Am Soc Nephrol 28: 2961-2972, 2017.

21. Berthier CC, Bethunaickan R, Gonzalez-Rivera T, Nair V, Ramanujam M, Zhang W, Bottinger EP, Segerer S, Lindenmeyer M, Cohen CD, et al: Cross-species transcriptional network analysis defines shared inflammatory responses in murine and human lupus nephritis. J Immunol 189: 988-1001, 2012.

22. Shved N, Warsow G, Eichinger F, Hoogewijs D, Brandt S, Wild P, Kretzler M, Cohen CD and Lindenmeyer MT: Transcriptome-based network analysis reveals renal cell type-specific dysregulation of hypoxia-associated transcripts. Sci Rep 7: 8576, 2017.

23. Gautier L, Cope L, Bolstad BM and Irizarry RA: Affy-analysis of affymetrix GeneChip data at the probe level. Bioinformatics 20: 307-315, 2004

24. Forero DA, Guio-Vega GP and Gonzalez-Giraldo Y: A comprehensive regional analysis of genome-wide expression profiles for major depressive disorder. J Affect Disord 218: 86-92, 2017

25. Leek JT, Johnson WE, Parker HS, Jaffe AE and Storey JD: The sva package for removing batch effects and other unwanted variation in high-throughput experiments. Bioinformatics 28 : $882-883,2012$

26. Diboun I, Wernisch L, Orengo CA and Koltzenburg M: Microarray analysis after RNA amplification can detect pronounced differences in gene expression using limma. BMC Genomics 7: 252, 2006.

27. Yu G, Wang LG, Han Y and He QY: ClusterProfiler: An R package for comparing biological themes among gene clusters. OMICS 16: 284-287, 2012

28. Szklarczyk D, Morris JH, Cook H, Kuhn M, Wyder S, Simonovic M, Santos A, Doncheva NT, Roth A, Bork P, et al: The STRING database in 2017: Quality-controlled protein-protein association networks, made broadly accessible. Nucleic Acids Res 45: D362-D368, 2017.

29. Kohl M, Wiese S and Warscheid B: Cytoscape: Software for visualization and analysis of biological networks. Methods Mol Biol 696: 291-303, 2011.

30. Chin $\mathrm{CH}$, Chen SH, Wu HH, Ho CW, Ko MT and Lin CY: cytoHubba: Identifying hub objects and sub-networks from complex interactome. BMC Syst Biol 8 (Suppl 4): S11, 2014.

31. Kawai Y, Masutani K, Torisu K, Katafuchi R, Tanaka S, Tsuchimoto A, Mitsuiki K, Tsuruya K and Kitazono T: Association between serum albumin level and incidence of end-stage renal disease in patients with immunoglobulin A nephropathy: A possible role of albumin as an antioxidant agent. PLoS One 13: e0196655, 2018.

32. Park HJ, Kim JW, Cho BS and Chung JH: Association of FOS-like antigen 1 promoter polymorphism with podocyte foot process effacement in immunoglobulin A nephropathy patients. J Clin Lab Anal 28: 391-397, 2014.

33. Qiu LQ, Sinniah R and Hsu SI: Coupled induction of iNOS and p53 upregulation in renal resident cells may be linked with apoptotic activity in the pathogenesis of progressive IgA nephropathy. J Am Soc Nephrol 15: 2066-2078, 2004.

34. Boguslawska J, Kedzierska H, Poplawski P, Rybicka B, Tanski Z and Piekielko-Witkowska A: Expression of genes involved in cellular adhesion and extracellular matrix remodeling correlates with poor survival of patients with renal cancer. J Urol 195: 1892-1902, 2016.

35. Livingston MJ, Ding HF, Huang S, Hill JA, Yin XM and Dong Z: Persistent activation of autophagy in kidney tubular cells promotes renal interstitial fibrosis during unilateral ureteral obstruction. Autophagy 12: 976-998, 2016.

36. Glasner A, Levi A, Enk J, Isaacson B, Viukov S, Orlanski S, Scope A, Neuman T, Enk CD, Hanna JH, et al: NKp46 receptor-mediated interferon- $\gamma$ production by natural killer cells increases fibronectin 1 to alter tumor architecture and control metastasis. Immunity 48: 107-119.e4, 2018.

37. Jovanovic KK, Escure G, Demonchy J, Willaume A, Van de Wyngaert Z, Farhat M, Chauvet P, Facon T, Quesnel B and Manier S: Deregulation and targeting of TP53 pathway in multiple myeloma. Front Oncol 8: 665, 2019.
38. Yogosawa S and Yoshida K: Tumor suppressive role for kinases phosphorylating p53 in DNA damage-induced apoptosis. Cancer Sci 109: 3376-3382, 2018

39. Zhang SZ, Qiu XJ, Dong SS, Zhou LN, Zhu Y, Wang MD and Jin LW: MicroRNA-770-5p is involved in the development of diabetic nephropathy through regulating podocyte apoptosis by targeting TP53 regulated inhibitor of apoptosis 1. Eur Rev Med Pharmacol Sci 23: 1248-1256, 2019.

40. Chen SN, Lombardi R, Karmouch J, Tsai JY, Czernuszewicz G, Taylor MRG, Mestroni L, Coarfa C, Gurha P and Marian AJ: DNA damage Response/TP53 pathway is activated and contributes to the pathogenesis of dilated cardiomyopathy associated With LMNA (Lamin A/C) mutations. Circ Res 124: 856-873, 2019.

41. Liu GC, Zhou YF, Su XC and Zhang J: Interaction between TP53 and XRCC1 increases susceptibility to cervical cancer development: A case control study. BMC Cancer 19: 24, 2019.

42. Robin M, Issa AR, Santos CC, Napoletano F, Petitgas C, Chatelain G, Ruby M, Walter L, Birman S, Domingos PM, et al: Drosophila p53 integrates the antagonism between autophagy and apoptosis in response to stress. Autophagy 15: 771-784, 2019.

43. Dai J, Xu LJ, Han GD, Jiang HT, Sun HL, Zhu GT and Tang XM: Down-regulation of long non-coding RNA ITGB2-AS1 inhibits osteosarcoma proliferation and metastasis by repressing $\mathrm{Wnt} / \beta$-catenin signalling and predicts favourable prognosis. Artif Cells Nanomed Biotechnol 46 (Suppl 3): S783-S790, 2018.

44. Liu H, Dai X, Cao X, Yan H, Ji X, Zhang H, Shen S, Si Y, Zhang $\mathrm{H}$, Chen J, et al: PRDM4 mediates YAP-induced cell invasion by activating leukocyte-specific integrin $\beta 2$ expression. EMBO Rep 19: pii, e45180, 2018.

45. Aziz MH, Cui K, Das M, Brown KE, Ardell CL, Febbraio M, Pluskota E, Han J, Wu H, Ballantyne CM, et al: The upregulation of integrin $\alpha \mathrm{D} \beta 2(\mathrm{CD} 11 \mathrm{~d} / \mathrm{CD} 18)$ on inflammatory macrophages promotes macrophage retention in vascular lesions and development of atherosclerosis. J Immunol 198: 4855-4867, 2017.

46. Kijas JM, Bauer TR Jr, Gafvert S, Marklund S, Trowald-Wigh G, Johannisson A, Hedhammar A, Binns M, Juneja RK, Hickstein DD and Andersson L: A missense mutation in the beta-2 integrin gene (ITGB2) causes canine leukocyte adhesion deficiency. Genomics 61: 101-107, 1999.

47. Yee NK and Hamerman JA: $\beta(2)$ integrins inhibit TLR responses by regulating NF- $\mathrm{NB}$ pathway and $\mathrm{p} 38$ MAPK activation. Eur J Immunol 43: 779-792, 2013

48. Duan J, Zhang X, Zhang S, Hua S and Feng Z: miR-206 inhibits FN1 expression and proliferation and promotes apoptosis of rat type II alveolar epithelial cells. Exp Ther Med 13: 3203-3208, 2017.

49. Wang J, Deng L, Huang J, Cai R, Zhu X, Liu F, Wang Q, Zhang J and Zheng Y: High expression of Fibronectin 1 suppresses apoptosis through the NF- $\kappa \mathrm{B}$ pathway and is associated with migration in nasopharyngeal carcinoma. Am J Transl Res 9: 4502-4511, 2017.

50. Liu C, Feng Z, Chen T, Lv J, Liu P, Jia L, Zhu J, Chen F, Yang C and Deng Z: Downregulation of NEAT1 reverses the radioactive iodine resistance of papillary thyroid carcinoma cell via miR-101-3p/FN1/PI3K-AKT signaling pathway. Cell Cycle 18: 167-203, 2019.

51. Liao YX, Zhang ZP, Zhao J and Liu JP: Effects of Fibronectin 1 on cell proliferation, senescence and apoptosis of human glioma cells through the PI3K/AKT signaling pathway. Cell Physiol Biochem 48: 1382-1396, 2018.

52. Bakiri L, Hamacher R, Grana O, Guío-Carrión A, Campos-Olivas R, Martinez L, Dienes HP, Thomsen MK, Hasenfuss SC and Wagner EF: Liver carcinogenesis by FOS-dependent inflammation and cholesterol dysregulation. J Exp Med 214: 1387-1409, 2017.

53. Xu X, Kwon OK, Shin IS, Mali JR, Harmalkar DS, Lim Y, Lee G, Lu Q, Oh SR, Ahn KS, et al: Novel benzofuran derivative DK-1014 attenuates lung inflammation via blocking of MAPK/AP-1 and AKT/mTOR signaling in vitro and in vivo. Sci Rep 9: 862, 2019.

54. Chen M, Li X, Shi Q, Zhang Z and Xu S: Hydrogen sulfide exposure triggers chicken trachea inflammatory injury through oxidative stress-mediated FOS/IL8 signaling. J Hazard Mater 368: 243-254, 2019.

55. Nagesh R, Kiran Kumar KM, Naveen Kumar M, Patil RH and Sharma SC: Stress activated p38 MAPK regulates cell cycle via AP-1 factors in areca extract exposed human lung epithelial cells. Cytotechnology 71: 507-520, 2019. 
56. Wu DM,Zhang YT,Lu J and Zheng YL: Effects of microRNA-129 and its target gene c-Fos on proliferation and apoptosis of hippocampal neurons in rats with epilepsy via the MAPK signaling pathway. J Cell Physiol 233: 6632-6643, 2018.

57. Liu C, Ding L, Bai L, Chen X, Kang H, Hou L and Wang J: Folate receptor alpha is associated with cervical carcinogenesis and regulates cervical cancer cells growth by activating ERK1/2/ c-Fos/c-Jun. Biochem Biophys Res Commun 491: 1083-1091, 2017.

58. Hwang MS, Strainic MG, Pohlmann E, Kim H, Pluskota E, Ramirez-Bergeron DL, Plow EF and Medof ME: VEGFR2 survival and mitotic signaling depends on joint-activation of associated C3ar1/C5ar1 and IL-6R-gp130. J Cell Sci 132: pii: jcs219352, 2019

59. Mathern DR, K Horwitz J and Heeger PS: Absence of recipient C3aR1 signaling limits expansion and differentiation of alloreactive $\mathrm{CD} 8^{+} \mathrm{T}$ cell immunity and prolongs murine cardiac allograft survival. Am J Transplant 198: 1628-1640, 2019.

60. Matsumoto N, Satyam A, Geha M, Lapchak PH, Dalle Lucca JJ, Tsokos MG and Tsokos GC: C3a enhances the formation of intestinal organoids through C3aR1. Front Immunol 8: 1046, 2017.

61. Lokman FE, Seman NA, Ismail AA, Yaacob NA, Mustafa N, Khir AS, Hussein Z and Wan Mohamud WN: Gene expression profiling in ethnic Malays with type 2 diabetes mellitus, with and without diabetic nephropathy. J Nephrol 24: 778-789, 2011.

62. Wang D, Guan MP, Zheng ZJ, Li WQ, Lyv FP, Pang RY and Xue YM: Transcription factor Egrl is involved in high glucose-induced proliferation and fibrosis in rat glomerular mesangial cells. Cell Physiol Biochem 36: 2093-2107, 2015

63. Liu F, Zhang ZP, Xin GD, Guo LH, Jiang Q and Wang ZX: miR-192 prevents renal tubulointerstitial fibrosis in diabetic nephropathy by targeting Egrl. Eur Rev Med Pharmacol Sci 22: 4252-4260, 2018.

64. Wu C, Ma X, Zhou Y, Liu Y, Shao Y and Wang Q: Klotho restraining Egr1/TLR4/mTOR axis to reducing the expression of fibrosis and inflammatory cytokines in high glucose cultured rat mesangial cells. Exp Clin Endocrinol Diabetes: Jun 11, 2018 (Epub ahead of print). doi: 10.1055/s-0044-101601.

65. Hu F, Xue M, Li Y, Jia YJ, Zheng ZJ, Yang YL, Guan MP, Sun L and Xue YM: Early growth response 1 (Egr1) is a transcriptional activator of NOX4 in oxidative stress of diabetic kidney disease. J Diabetes Res 2018: 3405695, 2018

66. Li Y, Hu F, Xue M, Jia YJ, Zheng ZJ, Wang L, Guan MP and Xue YM: Klotho down-regulates Egr-1 by inhibiting TGF- $\beta 1 /$ Smad3 signaling in high glucose treated human mesangial cells. Biochem Biophys Res Commun 487: 216-222, 2017.

67. Wu SY, Rupaimoole R, Shen F, Pradeep S, Pecot CV, Ivan C Nagaraja AS, Gharpure KM, Pham E, Hatakeyama H, et al: A miR-192-EGR1-HOXB9 regulatory network controls the angiogenic switch in cancer. Nat Commun 7: 11169, 2016.

68. Sun S, Ning X, Zhai Y, Du R, Lu Y, He L, Li R, Wu W, Sun W and Wang H: Egr-1 mediates chronic hypoxia-induced renal interstitial fibrosis via the PKC/ERK pathway. Am J Nephrol 39: 436-448, 2014

69. Lin CY,Lin TY, Lee MC, Chen SC and Chang JS: Hyperglycemia: GDNF-EGR1 pathway target renal epithelial cell migration and apoptosis in diabetic renal embryopathy. PLoS One 8: e56731, 2013.

70. Singh R, Yadav V, Kumar S and Saini N: MicroRNA-195 inhibits proliferation, invasion and metastasis in breast cancer cells by targeting FASN, HMGCR, ACACA and CYP27B1. Sci Rep 5: 17454,2015

71. Banks M and Holick MF: Molecular mechanism(s) involved in 25-hydroxyvitamin D's antiproliferative effects in CYP27B1-transfected LNCaP cells. Anticancer Res 35: $3773-3779,2015$
72. Li G, Wu F, Yang H, Deng X and Yuan Y: MiR-9-5p promotes cell growth and metastasis in non-small cell lung cancer through the repression of TGFBR2. Biomed Pharmacother 96: 1170-1178, 2017.

73. Ebefors K, Liu P, Lassén E, Elvin J, Candemark E, Levan K, Haraldsson B and Nyström J: Mesangial cells from patients with IgA nephropathy have increased susceptibility to galactose-deficient IgA1. BMC Nephrol 17: 40, 2016.

74. You Z, Xu J, Li B, Ye H, Chen L, Liu Y and Xiong X: The mechanism of ATF3 repression of epithelial-mesenchymal transition and suppression of cell viability in cholangiocarcinoma via p53 signal pathway. J Cell Mol Med 23: 2184-2193, 2019.

75. Hsu YJ, Hsu SC, Chang YL, Huang SM, Shih CC, Tsai CS and Lin CY: Indoxyl sulfate upregulates the cannabinoid type 1 receptor gene via an ATF3/c-Jun complex-mediated signaling pathway in the model of uremic cardiomyopathy. Int J Cardiol 252: 128-135, 2018.

76. Armstrong HK, Gillis JL, Johnson IRD, Nassar ZD, Moldovan M, Levrier C, Sadowski MC, Chin MY, Tomlinson Guns ES, Tarulli G, et al: Dysregulated fibronectin trafficking by Hsp90 inhibition restricts prostate cancer cell invasion. Sci Rep 8: 2090, 2018.

77. Wei X, Zhou D, Wang H, Ding N, Cui XX, Wang H, Verano M, Zhang K, Conney AH, Zheng X and DU ZY: Effects of pyridine analogs of curcumin on growth, apoptosis and NF- $\mathrm{KB}$ activity in prostate cancer PC-3 cells. Anticancer Res 33: 1343-1350, 2013.

78. Bork P: The modular architecture of a new family of growth regulators related to connective tissue growth factor. FEBS Lett 327: 125-130, 1993.

79. Chen CC and Lau LF: Functions and mechanisms of action of CCN matricellular proteins. Int J Biochem Cell Biol 41: 771-783, 2009.

80. Quan T, He T, Shao Y, Lin L, Kang S, Voorhees JJ and Fisher GJ: Elevated cysteine-rich 61 mediates aberrant collagen homeostasis in chronologically aged and photoaged human skin. Am J Pathol 169: 482-490, 2006

81. Mizwicki MT, Liu G, Fiala M, Magpantay L, Sayre J, Siani A, Mahanian M, Weitzman R, Hayden EY, Rosenthal MJ, et al: 1 $\alpha, 25$-dihydroxyvitamin D3 and resolvin D1 retune the balance between amyloid- $\beta$ phagocytosis and inflammation in Alzheimer's disease patients. J Alzheimers Dis 34: 155-170, 2013.

82. Carl M, Akagi Y, Weidner S, Isaka Y, Imai E and Rupprecht HD: Specific inhibition of Egr-1 prevents mesangial cell hypercellularity in experimental nephritis. Kidney Int 63: 1302-1312, 2003.

83. Mohamad T, Kazim N, Adhikari A and Davie JK: EGR1 interacts with TBX2 and functions as a tumor suppressor in rhabdomyosarcoma. Oncotarget 9: 18084-18098, 2018.

84. Carver KA, Smith TL, Gallagher PE and Tallant EA Angiotensin-(1-7) prevents angiotensin II-induced fibrosis in cremaster microvessels. Microcirculation 22: 19-27, 2015.

85. Heider A and Alt R: virtualArray: A R/bioconductor package to merge raw data from different microarray platforms. BMC Bioinformatics 14: 75, 2013

86. Tokunaga K, Uto H, Takami Y, Mera K, Nishida C, Yoshimine Y, Fukumoto M, Oku M, Sogabe A, Nosaki T, et al: Insulin-like growth factor binding protein-1 levels are increased in patients with IgA nephropathy. Biochem Biophys Res Commun 399: 144-149, 2010.

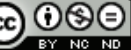

This work is licensed under a Creative Commons Attribution-NonCommercial-NoDerivatives 4.0 International (CC BY-NC-ND 4.0) License. 